\title{
Beneficial effect of real-time continuous glucose monitoring system on glycemic control in type 1 diabetic patients: systematic review and meta-analysis of randomized trials
}

\author{
A Szypowska, A Ramotowska, K Dżygało and D Golicki ${ }^{1}$ \\ Department of Pediatrics, Medical University of Warsaw, Dzialdowska 1, 01-184 Warsaw, Poland and ${ }^{1}$ Department of Pharmacoeconomics, Medical \\ University of Warsaw, Warsaw, Poland \\ (Correspondence should be addressed to A Szypowska; Email: agnieszka.szypowska@gmail.com)
}

\begin{abstract}
Objective: Real-time continuous glucose monitoring (RT-CGM) provides detailed information on glucose patterns and trends, thus allowing the patients to manage their diabetes more effectively.

Design: The aim of this study was to explore the potential beneficial effects of the use of RT-CGM on diabetes management compared with self blood glucose measurement (SBGM) in patients with type 1 diabetes mellitus (T1DM), by means of a systematic review and meta-analysis of randomized controlled trials (RCTs). Methods: MEDLINE, EMBASE, and the Cochrane Library were searched through by two independent investigators for RCTs concerning the use of RT-CGM in patients with T1DM. Only studies with a similar insulin regimen in the experimental and control groups were included in the analysis.

Results: Seven RCTs $(n=948)$ met the inclusion criteria. Combined data from all studies showed better $\mathrm{HbA1c}$ reduction in subjects using RT-CGM compared with those using SBGM (mean difference (MD) $-0.25 ; 95 \%$ confidence interval $(95 \% \mathrm{CI})$ : from -0.34 to $-0.17 ; P<0.001)$. Patients treated with insulin pump and RT-CGM had a lower HbA1c level compared with subjects managed with insulin pump and SBGM (four RCTs, $n=497$; MD -0.26 ; $95 \%$ CI: from -0.43 to $-0.10 ; P=0.002$ ). The benefits of applying RT-CGM were not associated with an increasing rate of major hypoglycemic episodes. The use of RT-CGM for over $60-70 \%$ of time was associated with a significant lowering of HbA1c.

Conclusions: RT-CGM is more beneficial than SBGM in reducing HbA1c in patients with type 1 diabetes. Further studies are needed to evaluate the efficacy of this system in the pediatric population, especially in very young children.
\end{abstract}

European Journal of Endocrinology 166 567-574

\section{Background}

The Diabetes Control and Complications Trial confirmed that tight metabolic control is regarded as crucial to prevent microvascular and macrovascular complications in type 1 diabetic patients (1). Both HbA1c and glucose variability play important roles in the evaluation of the risk of long-term diabetic complications (2). Intensive insulin therapy prevents or at least delays long-term diabetic complications. Aggressive diabetes management with continuous subcutaneous insulin infusion (CSII) or multiple daily injections (MDI) using insulin analogs and frequent blood glucose monitoring are the recommended methods to achieve therapeutic targets in type 1 diabetic patients.

The main factor limiting insulin management of type 1 diabetes mellitus (T1DM) subjects in the achievement of a strict glycemic goal is hypoglycemia (3). Unfortunately, despite active education, it is quite difficult to avoid hypoglycemia. Even the most frequent self blood glucose measurement (SBGM) gives insufficient information. Usually, T1DM patients carry out between four and eight finger-prick measurements per day, or less, and rarely monitor their blood glucose level at night. This is the cause of overlooking blood glucose excursion, and especially postprandial hyperglycemia, asymptomatic hypoglycemia, and glucose fluctuation during the night.

Continuous glucose monitoring (CGM) provides detailed information on glucose patterns and trends, thus allowing patients to manage their diabetes more effectively. Several continuous monitoring systems are commercially available. Some of them use CGM in a retrospective way and others are real-time glucose monitors. There are different types of real-time glucose monitors: the DexCom Seven (DexCom, San Diego, CA, USA), the MiniMed Paradigm Real-time Insulin Pump and CGM System (Medtronic, Medtronic MiniMed, Northridge, CA, USA), and the FreeStyle Navigator 
(Abbott Diabetes Care, Abbott Park, IL, USA). Each system consists of a glucose oxidase-based electrochemical sensor, which is placed subcutaneously and replaced every 3-7 days. Interstitial glucose measurements are sent continuously from the sensor to a receiver through advanced radio frequency wireless technology (4).

According to our previous meta-analysis, randomized controlled trials (RCTs) using CGMS in a retrospective way compared with SBGM did not show significant reduction in HbAlc in type 1 diabetic patients $(5,6)$. Real-time CGM (RT-CGM) provides a new dimension to diabetes management. Several studies, many of them observational, have assessed the effect of RT-CGM on metabolic control in type 1 diabetic patients (7). A number of trials have demonstrated a reduction in HbA1c with RT-CGM. Other studies have not confirmed any benefits or have found that the benefit associated with CGM was strongly related to age.

In this study, we sought to explore the potential beneficial effects of the use of RT-CGM on diabetes management compared with SBGM in patients with type 1 diabetes, by conducting a systematic review and meta-analysis of RCTs.

\section{Inclusion and exclusion criteria}

The systematic review and meta-analysis were conducted according to standards of the Cochrane Collaboration (8). Studies included in the review had to be RCTs with parallel or crossover design in which RT-CGM and self-monitoring of blood glucose were compared with self-monitoring of blood glucose alone in the management of type 1 diabetes. We included studies that used commercially available real-time glucose monitors: the DexCom Seven (DexCom), the MiniMed Paradigm Real-time Insulin Pump and CGM System (Medtronic), or the FreeStyle Navigator (Abbott Diabetes Care) and Guardian RT (Medtronic MiniMed, Northridge, CA, USA). Each system consists of a glucose oxidase-based electrochemical sensor, which is placed subcutaneously and along with a receiver to which interstitial glucose measurements are sent wirelessly and stored. A significant benefit of CSII over MDI for $\mathrm{HbAlc}$ reduction had been previously confirmed by some authors. Therefore, only studies with the same insulin regimen or studies with a similar proportion of patients using CSII and MDI in both experimental and control groups were included in the analysis. The studies had to be of at least 3 months' duration and had to have a follow-up rate of over $80 \%$. We excluded unpublished studies, letters to the editor, abstracts, and proceedings of scientific meetings. We also excluded studies in which patients used both CSII and MDI, but in which authors gave no information about the structure of usage in the experimental and control groups or the groups were not balanced in terms of the usage structure. We also excluded trials involving patients with type 2 diabetes, pregnant women with
T1DM, and pancreas/islet-cell transplant patients. Studies using the Gluco-Watch G2 Biographer (Cygnus, Redwood City, CA, US) were not included in this analysis due to a different method of glucose measurement. This device takes non-invasive glucose measurements using low electric current to pull glucose through the skin. It caused a lot of skin irritations that led to very low compliance rates. Moreover, because of its side effects, the Gluco-Watch G2 Biographer has been withdrawn from the market. Trials that used other RT-CGM devices that are not available on the market anymore, or evaluated the use of blinded, retrospective CGM were excluded. Studies performed in settings such as pre- and post-surgical or cardiac care unit were excluded as well.

\section{Outcomes}

The primary end point was the change in $\mathrm{HbAlc}$ between the RT-CGM and the SBGM groups. The secondary end points were: major and minor hypoglycemic episodes (as defined by the investigators), mean daily area under the CGM curve for glucose $<3.89 \mathrm{mmol} / \mathrm{l}$, mean daily area over the CGM curve for glucose $>9.99 \mathrm{mmol} / \mathrm{l}$, local adverse effects and quality of life (QoL).

\section{Search strategy}

The following electronic databases were systematically searched through for relevant studies: MEDLINE (PubMed), EMBASE (Ovid), and the Cochrane Central Register of Controlled Trials. The search was conducted from 1996 to March 2011. The search strategy included the use of a validated filter for identifying RCTs (9). Keywords included a constellation of different phrases centered around CGM system ('CGMS' or 'CGM' or 'Continuous Glucose Monitoring' or 'continuous glucose monitoring' or 'RT-CGM' or 'continuous subcutaneous glucose monitoring' or 'DexCom' or 'real-time system' or 'FreeStyle Navigator' or 'guardian' or 'sensor-augmented insulin pump') and type 1 diabetes ('diabetes type 1' or 'diabetes t. 1 ' or 'diabetes mellitus' or 'juvenile onset' or 'type 1 diabetes' or 'IDDM' or 'autoimmune diabetes' or 'DM1' or 'DM type 1 ' or 'insulin-dependent' or 'T1DM' or 'brittle diabetes' or 'T1D'). Subsequently, reference lists based on original studies and review articles were identified.

\section{Data extraction}

Two independent reviewers (A Ramotowska and $\mathrm{K}$ Dżygało) screened the abstracts from the clinical trials according to the search strategy. Full texts of all potentially relevant articles were examined to determine whether they met the inclusion criteria. Both reviewers (A Ramotowska and K Dżygało) extracted data independently, using standard data extraction forms. Extracted data were compared to 
eliminate errors. All disagreements between the reviewers were resolved by consensus, or if the consensus was not reached, by a third reviewer (A Szypowska).

\section{Study quality}

The methodological quality of the included studies was assessed by independent reviewers, without blinding to authorship or journal. The application of the following strategies associated with good-quality studies was examined: i) allocation concealment; ii) blinding of participants, investigators, outcome assessors and data analysts (yes/no); iii) intention-to-treat (ITT) analysis (yes/no); and iv) comprehensive follow-up. The allocation concealment was considered adequate when the randomization method used did not allow the investigator or the participant to identify or influence the intervention group before the entry of eligible participants into the study. The quality of allocation concealment was regarded as unclear when randomization was used, but no information about the method of randomization was available. It was regarded as inadequate when inappropriate methods of randomization (e.g. alternate medical record numbers, unsealed envelopes and tossing the coin) were used. In ITT analysis, a 'yes' answer meant that the authors had specifically reported undertaking this type of analysis and/or that our own study confirmed this finding. Conversely, 'no' meant that the authors had not reported the use of ITT analysis and/or that we could not confirm its use in the study assessment. The completeness of patient follow-up was evaluated by ascertaining the percentage of participants excluded or lost to follow-up. Completeness of follow-up was considered to be adequate if $\geq 80 \%$ of participants were included in the final analysis.

\section{Statistical analysis}

We used data from the end of each trial included in the systematic review. Data were analyzed using Comprehensive Meta-analysis Software (version 2.2.057; Biostat, Englewood, NJ, USA) (10). The mean difference (MD) was selected to determine differences in continuous outcomes between the experimental and control groups. The binary measure for individual studies and pooled statistics was calculated as the risk ratio (RR) between the experimental and the control groups, with 95\% confidence interval $(95 \% \mathrm{CI})$. The difference between the study groups was considered significant when the $P$ value was $<0.05$ or when the $95 \%$ CI for RR did not exceed 1.0 and that for MD did not exceed 0 . Heterogeneity was determined by $I^{2}$. Substantial heterogeneity was represented by $\mathrm{I}^{2}$ of $50 \%$ or more (11). A fixed-effect model was used as baseline and a random-effect model was used in case of substantial heterogeneity.

\section{Results}

\section{Study description}

Based on the search strategy, 744 abstracts from clinical trials regarding CGMS were identified. The diagram of data extraction is illustrated in Fig. 1. We identified 38 articles that underwent further analysis. Finally, we included seven RCTs $(n=948)$ to both qualitative and quantitative analyses $(12,13,14,15,16,17,18)$. Table 1 summarizes the characteristics of the included trials. In five studies, insulin pump therapy was used in both experimental and control groups $(13,14,16,17$, $18)$; in the next two studies, the number of patients treated with CSII or MDI was comparable for the experimental and control groups $(12,15)$. All trials included in the review, except one (17), were multicentered. All trials contained a sufficient proportion $(\geq 80 \%)$ of participants in the final analysis. One of them included only a pediatric population (16), one regarded only adults (17), and the rest assessed mixed populations. The follow-up period ranged from 3 to 12 months. In four studies, randomization sequences were described and were adequate $(12,15,16,18)$. Allocation concealment was well reported and suitable in two studies $(16,18)$. Investigators of two studies conducted ITT analyses $(12,15)$. Withdrawals and dropouts were described in two studies $(13,18)$. Table 2 summarizes the quality assessment of the included studies.

\section{HbA1c}

Meta-analysis of seven RCTs (948 subjects) showed a significant reduction in $\mathrm{HbA1c}(\mathrm{MD}-0.25 ; 95 \%$ CI: from -0.34 to $-0.17 ; P<0.001)$ for patients managed with RT-CGMS compared with patients monitored with SBGM (Fig. 2). Moreover, patients treated with insulin pump combined with RT-CGM had a lower HbA1c level (four RCTs, $n=497$; MD - 0.26; 95\% CI: from -0.43 to $-0.10 ; P=0.002$ ) compared with subjects managed with conventional insulin pump combined with SBGM (Fig. 3). The reduction

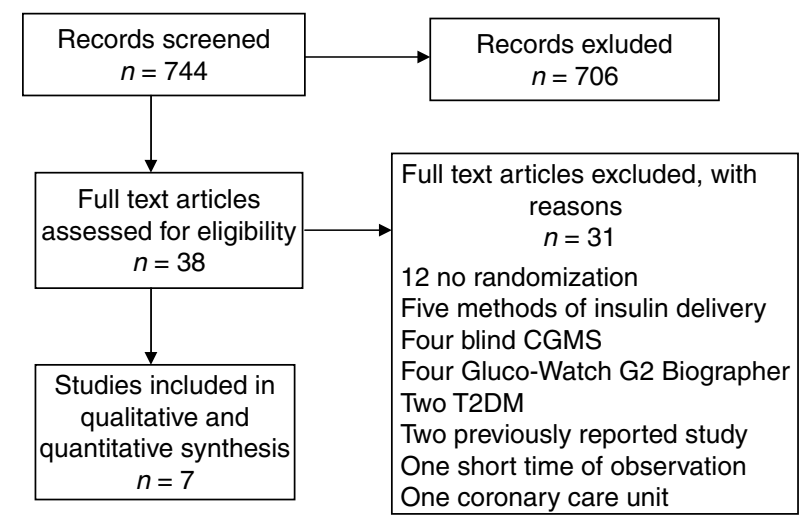

Figure 1 Diagram of data extraction. 


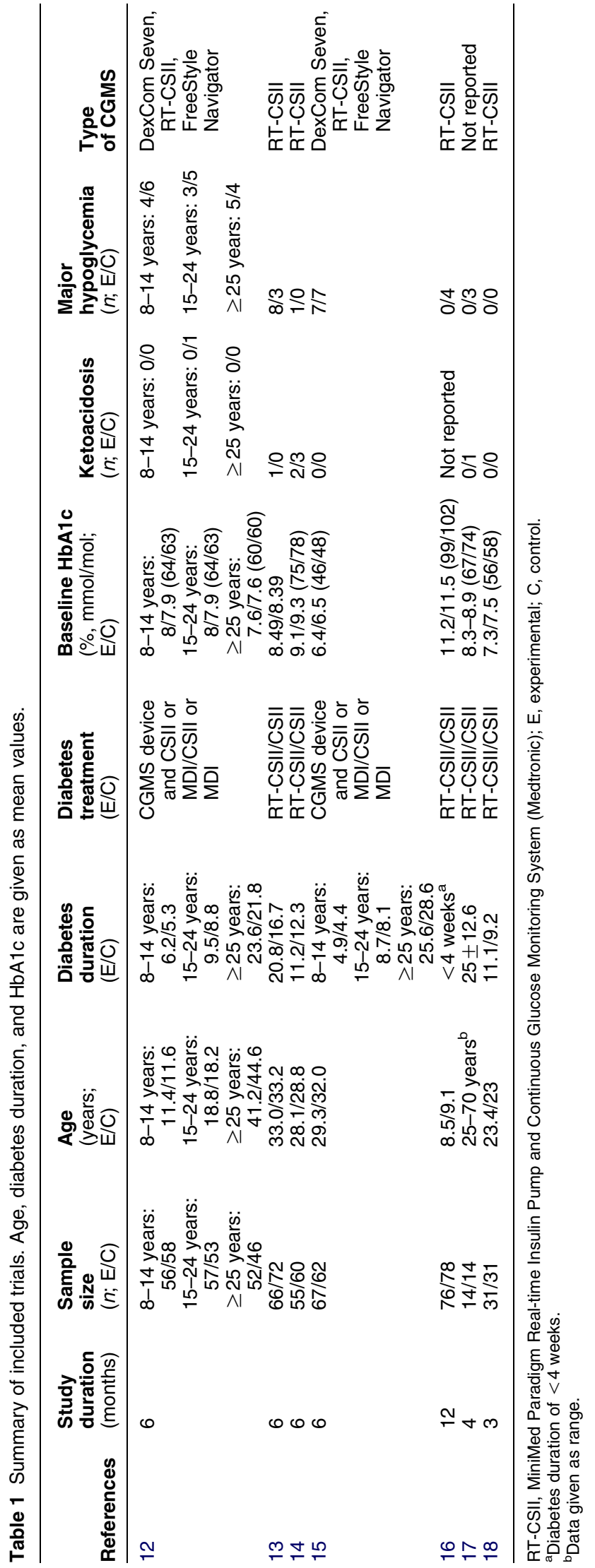

in HbAlc in adults (three RCTs, $n=224$, MD -0.37 ; $95 \%$ CI: from -0.76 to $0.02 ; P=0.06, I^{2}=77 \%$ ) and in children (three RCTs, $n=308$, MD $-0.19 ; 95 \%$ CI: from -0.42 to $-0.03 ; P=0.09)$ using RT-CGM compared with SBGM groups was close to statistical significance. An additional analysis in subgroups divided according to glycemic control showed lower HbAlc in patients managed with RT-CGM compared with those managed with SBGM in both subgroups: with good metabolic control (one RCT, $n=129$, MD $-0.31 ; 95 \%$ CI: from -0.46 to $-0.16 ; P<0.001)$ and poor glycemic control (four RCTs, $n=603$, MD $-0.21 ; 95 \%$ CI: from -0.32 to $-0.09 ; P<0.001)$ at baseline.

There was a significant inverse correlation between the $\mathrm{HbA} 1 \mathrm{c}$ level and the frequency of sensor use (13, $14,16,18)$. In the JDRF study (12), RT-CGM effectively lowered $\mathrm{HbA} 1 \mathrm{c}$ only in adults aged $\geq 25$ years. In four studies, more subjects in the RT-CGM group achieved the level of $\mathrm{HbA} 1 \mathrm{c}$ of $\leq 7 \%(53 \mathrm{mmol} / \mathrm{mol})$ than in the SBGM group $(12,13,15,18)$.

\section{Major hypoglycemic episodes}

RT-CGM usage had no influence on the incidence of major hypoglycemic episodes (six RCTs, $n=864$, RR $0.69 ; 95 \%$ CI: $0.41-1.14 ; P=0.15)$. The data are shown in Fig. 4. None of the included studies confirmed that RT-CGM decreased the rate of major hypoglycemia. In two studies, authors excluded patients with a history of major hypoglycemia $(12,18)$.

\section{Minor hypoglycemic episodes}

Minor hypoglycemia, defined as glucose level below $3.89 \mathrm{mmol} / \mathrm{l}(70 \mathrm{mg} \%)$, was presented in five studies in two ways: as a number of episodes and as time spent in hypoglycemia $(12,13,14,15,18)$. In one of them, authors did not find any difference in hypoglycemic episodes between patients using RT-CGM and control groups (13). There was no significant reduction in time spent in hypoglycemia in RT-CGM subjects, compared with that in SBGM group $(12,14,15,18)$.

\section{Mean daily time and daily area under the CGM curve for glucose level of $<3.89 \mathrm{mmol} / \mathrm{I}$}

The area under the curve (AUC) calculated from CGM for glucose $<3.89 \mathrm{mmol} / \mathrm{l}(70 \mathrm{mg} \%)$ was significantly reduced in RT-CGM groups compared with patients monitored with SBGM in two studies $(13,15)$. Other authors did not show any differences between RT-CGM and control groups (14).

\section{Hyperglycemia >9.99 $\mathrm{mmol} / \mathrm{l}(180 \mathrm{mg} \%)$}

A significant difference in favor of the RT-CGM group was observed with respect to time spent in 
Table 2 Quality assessment of included studies.

\begin{tabular}{|c|c|c|c|c|c|c|}
\hline References & Randomization & $\begin{array}{l}\text { Allocation } \\
\text { concealment }\end{array}$ & ITT & Blinding & Design & Follow-up (\%) \\
\hline 12 & $\begin{array}{l}\text { Permuted-block design stratified according to } \\
\text { clinical center, age group, and } \mathrm{HbA} 1 \mathrm{c} \text { level }\end{array}$ & Not described & Yes & No & Parallel & 98 \\
\hline 13 & Not described & Not described & No & No & Parallel & 95 \\
\hline 14 & Not described & Not described & No & No & Parallel & 87 \\
\hline 15 & $\begin{array}{l}\text { Permuted-block design stratified according to } \\
\text { clinical center, age group, and } \mathrm{HbA} 1 \mathrm{c} \text { level }\end{array}$ & Not described & Yes & No & Parallel & 98 \\
\hline 16 & Central randomization procedure & Yes & No & No & Parallel & 96 \\
\hline 17 & Not described & Not described & No & No & Parallel & 96 \\
\hline 18 & Central computer-generated schedule & Yes & No & No & Parallel & 89 \\
\hline
\end{tabular}

hyperglycemia in two studies $(12,14)$, which was not confirmed by other authors $(15,18)$. In two studies, there was no difference between RT-CGM groups and controls in the number of hyperglycemic events (12, 13). A significantly lower AUC in the RT-CGM groups compared with controls was noted by some authors (14) and not by others (13). In addition, there was a significant reduction in episodes of glucose above $250 \mathrm{mg} \%$ in the RT-CGM group compared with controls in one study (12), which was not noted by other authors (15).

\section{Mean amplitude of glycemic excursions}

In two studies $(14,16)$, glycemic variability was significantly lower in the sensor group. The difference between the groups was not observed by other authors (15).

\section{Ketoacidosis and local adverse events}

Ketoacidosis was infrequent and without any significant difference between experimental and control groups. Local adverse events were uncommon and included mainly skin problems at the sensor or insulin infusion site.

\section{Compliance}

The sensor use was consistently high but declined over time in some trials $(12,14,15,16)$. An increased frequency of sensor use was associated with a greater reduction in $\operatorname{HbA1c}(13,14,15,16,18)$. The compliance with the sensor wear was age related and lower in children and the lowest in adolescents $(12,15)$. Self-reported prestudy daily blood glucose measurements were associated with successful use of RT-CGM (15). An association between sensor use and baseline $\mathrm{HbA1c}$ was not noted (12). No significant effect of age, duration of diabetes or duration of insulin pump therapy on the frequency of sensor use was noted by other authors (18).

\section{Quality of life}

Two studies $(16,17)$ estimated QoL as their secondary end points. We did not include this in our meta-analysis because of the different forms of evaluation used. In the trial by Kordonouri et al. (16), children aged 8-18 years and their primary caregivers were asked at the start of the study and at 24 and 52 weeks to complete the DISABKIDS and KIDSCREEN-27 questionnaires for evaluation of the patient's health-related QoL and their caregiver's impression of the patient's QoL. Own well-being was assessed with the WHO-5 questionnaire. For physical, psychological, social support, and school, the scores were significantly lower at baseline compared with European norm data, reached normal values after 6 months and remained normal after 1 year, with no differences between experimental and control groups.

In the study by Peyrot et al. (17) all participants completed the User Acceptance Questionnaire, Insulin Delivery System Rating Questionnaire, and Blood Glucose Monitoring System Rating Questionnaire, which was developed for this study. In this trial, the investigators found that several patient-reported outcomes were significantly more positive in the RT-CGM arm than the control arm, including satisfaction measures, particularly the burden of blood glucose monitoring and convenience, as well as measures of health-related QoL, including social burden and diabetes-related worries.

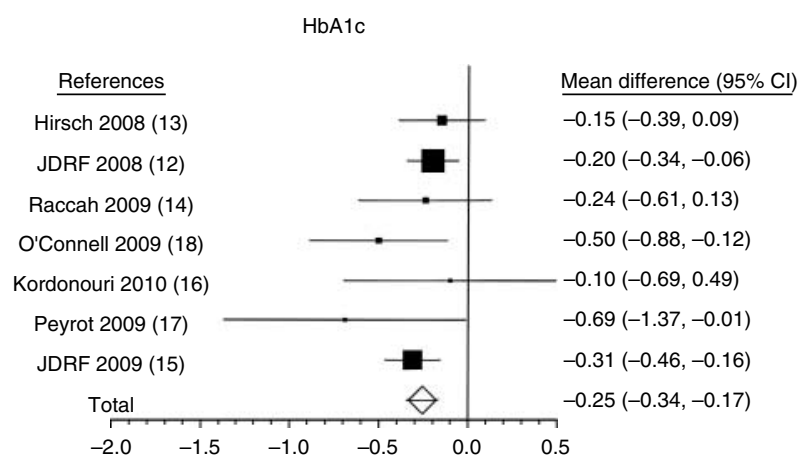

Figure 2 Mean difference and $95 \% \mathrm{Cl}$ of change in $\mathrm{HbA1c}(\%)$ of patients treated with CSII or MDI in whom the RT-CGM and SBGM were compared with SBGM alone in the management of T1DM. Fixed-effect model. Heterogeneity $R=0 \%$. 


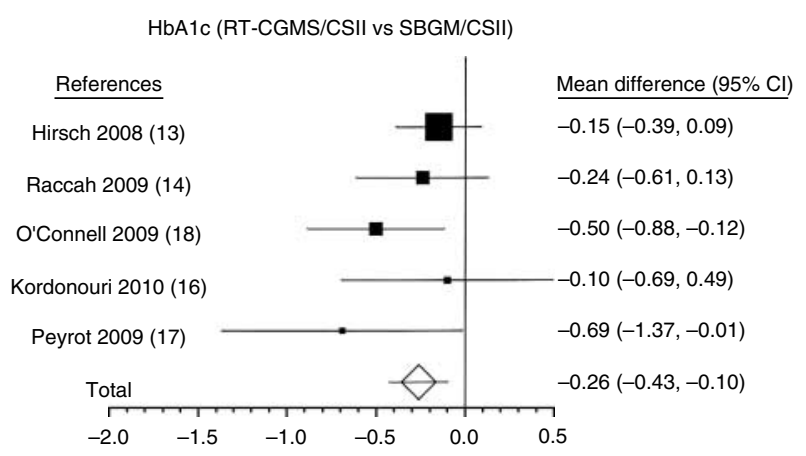

Figure 3 Mean difference and $95 \% \mathrm{Cl}$ of change in $\mathrm{HbA} 1 \mathrm{c}(\%)$ of patients managed with insulin pump combined with RT-CGM and SBGM in comparison with subjects treated with insulin pump and monitored with SBGM alone. Fixed-effect model. Heterogeneity $I^{2}=4 \%$.

\section{Discussion}

This meta-analysis of seven RCTs showed that the RT-CGM provides a superior benefit over self-monitoring of blood glucose with regard to $\mathrm{HbA1c}$ reduction in type 1 diabetic patients. The improvement in $\mathrm{HbA} 1 \mathrm{c}$ in patients using the RT-CGM was achieved without an increase in severe hypoglycemia.

The recently published systematic review of nine RCTs indicated that RT-CGM has a beneficial effect on glycemic control in adult patients with T1DM, without an increase in the incidence of hypoglycemia. Less convincing evidence was available for children and type 2 diabetes patients (19). The authors of this review could not perform a meta-analysis because of an extensive clinical heterogeneity of trials. They included in their analysis patients using different methods of insulin administration (MDI or CSII), with different types of diabetes (type 1 and/or type 2 diabetes), as well as subjects monitored with Gluco-Watch G2 Biographer. Our meta-analysis differs from the study by Hoeks et al. (19) due to different inclusion criteria. In our meta-analysis, we included only trials with a similar method of insulin administration in both control and experimental groups. Previous meta-analyses had already shown that CSII compared with MDI was a more effective form of metabolic control $(20,21)$. Therefore, assessment of the efficacy of RT-CGM is not possible if the insulin delivery method is different in experimental and control groups.

\section{Limitations at study and outcome level}

In all included trials, medical devices for real-time glucose measurement were used, therefore blinding was not possible. Some of the analyzed trials revealed methodological limitations, including the lack of ITT analysis, unclear or inadequate allocation concealments and no data describing randomization. In one study, the sample size was limited (17). Moreover, the trials were conducted for up to 12 months; most of them were carried out for a period of 3 or 6 months. The short duration of the follow-up made it difficult to predict whether the decreased $\mathrm{HbA} 1 \mathrm{c}$ level would be maintained for a longer period. In view of a marked heterogeneity in the definition and assessment of hypoglycemia, a pooled analysis of this end point was not performed. Some studies reported a positive association between the primary end point and the degree of compliance. However, QoL was only assessed in two studies (16, 17). These studies were conducted using different questionnaires. The lack of standard QoL questionnaires prevented execution of the analysis. We observed a substantial clinical heterogeneity of the analyzed studies performed in adults. To deal with the statistical heterogeneity, we used the random-effect model.

\section{Clinical implications}

The previous meta-analysis comparing blinded CGM with SBGM showed no superiority of CGM over SBGM in lowering $\mathrm{HbA} 1 \mathrm{c}$ in type 1 diabetic patients (5). However, those devices were clinician oriented and allowed only for a retrospective evaluation of data. A new generation of CGM devices offers real-time interstitial glucose monitoring and allows for advanced decisions made by patients. The results of our meta-analysis support the notion that the use of RT-CGM is associated with a significant lowering of $\mathrm{HbAlc}$ as well as glycemic variability. Both components - chronic sustained hyperglycemia and acute glycemic fluctuations - lead to diabetes complications through two main mechanisms excessive protein glycation and activation of oxidative stress (22). Tight glycemic control is therefore of great importance in diabetes management. According to ISPAD guidelines, a target range of $\mathrm{HbA} 1 \mathrm{c}$ for all age groups with type 1 diabetes of $<7.5 \%(58 \mathrm{mmol} / \mathrm{mol})$ is recommended (23). However, lowering HbA1c to below or around $7 \%(53 \mathrm{mmol} / \mathrm{mol})$ has been shown to reduce microvascular and neuropathic complications of diabetes. Therefore, in American Diabetes Association (ADA) recommendations, a reasonable $\mathrm{HbAlc}$ goal for many

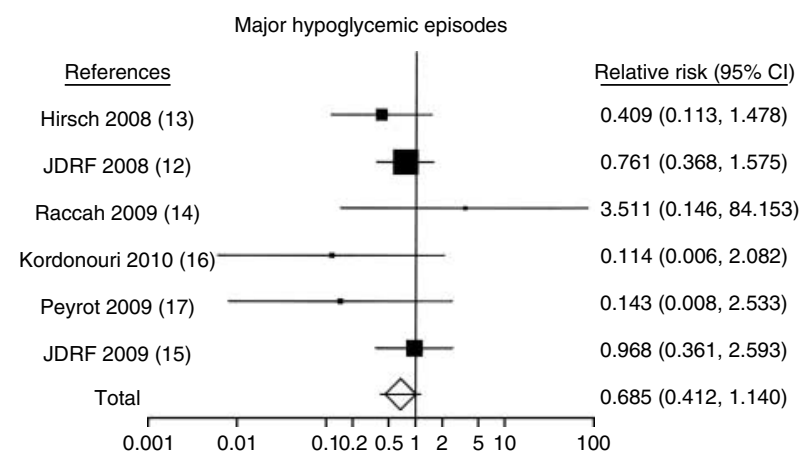

Figure 4 Forest plot showing the effect of RT-CGM and SBGM compared with that of SBGM on the proportion of patients with major hypoglycemic episodes at the end of the studies. $95 \% \mathrm{Cl}$, $95 \%$ confidence interval. Fixed-effect model. Heterogeneity $P=0 \%$. 
non-pregnant adults is $<7 \%$ (24). Our analysis showed that more patients in the RT-CGM group reached the target HbA1c of $7 \%$ or less $(12,13,15,18)$. Lower HbA1c values in the group using RT-CGM were not associated with an increased frequency of major hypoglycemic events. This not only reflects the benefits of RT-CGM, but also indicates the safety and efficacy of insulin analogs and insulin pumps. However, our results must be interpreted with caution since the included studies were not powered to evaluate the difference between the groups in terms of the rate of major hypoglycemia.

An important clinical question is which patients may benefit from RT-CGM use. Over $80 \%$ of patients included in our analysis were treated with CSII. Our previous meta-analysis demonstrated a statistical difference between CSII and MDI therapies (20). CSII therapy was associated with a significant reduction in $\mathrm{HbA} 1 \mathrm{c}$ in comparison to MDI, without an increased risk of major hypoglycemia. Our results showed that insulin pump used in combination with RT-CGM had a beneficial effect on glycemic control in T1DM subjects. Pump users managed with RT-CGM achieved significant lowering of $\mathrm{HbA} 1 \mathrm{c}$ in comparison with the subjects treated with conventional insulin pumps. Reduction in the HbAlc level in diabetic patients using RT-CGM was noted in participants with poor glycemic control. However, the study of T1DM subjects with good glycemic control confirmed the efficacy of RT-CGM in well-controlled diabetic patients as well. Our subanalyses of adults and children with type 1 diabetes did not show any beneficial effect of RT-CGM. However, the results, especially in adults, were close to statistical significance. The lack of superiority of RT-CGM over SBGM in lowering HbA1c might be partly a result of a small number of patients included in particular analyses. Another reason, especially in children, could be a low compliance.

Our meta-analysis showed that patients' motivation to use RT-CGM was crucial for device effectiveness. The most important factor influencing higher reduction in $\mathrm{HbA1c}$ was an increased frequency of sensor use. The use of RT-CGM for over $60-70 \%$ of time was associated with a significant lowering in $\operatorname{HbA1c}(13,14,15,16$, 18). Some authors noted a decline in sensor use over time. Moreover, comparing different age groups showed a lower compliance in children and the lowest compliance in teenagers. This shows that the currently available RT-CGM systems are not user-friendly enough, especially for children and their families.

\section{Implications for further research}

The use of RT-CGM provides a better insight into glycemic profiles, which may have a beneficial effect on patients with frequent severe hypoglycemia. Therefore, further studies are needed in subjects selected specifically for that problem. There are no randomized studies evaluating whether RT-CGM is beneficial in the management of toddlers and preschool children with T1DM. Although frequent SBGM is an integral part of intensive diabetes management, there are difficulties in minimizing glucose fluctuations in this age group. Parents and caregivers of young children experience a high level of stress related to fear of hypoglycemia, which can interfere with normal developmental and psychosocial interaction among diabetic children. Therefore, further studies are important not only for assessing the effectiveness, safety, and tolerance of RT-CGM device, but also to evaluate the impact of RT-CGM on the QoL. A decrease in compliance during the course of a trial was reported by some authors. Therefore, further research evaluating the lack or decreasing compliance in the follow-up is needed.

\section{Conclusions}

Our meta-analysis confirmed that the use of RT-CGM compared with SBGM effectively lowered HbA1c in type 1 diabetes. The benefit of applying RT-CGM was not associated with an increasing rate of acute hypoglycemia. The reduction in $\mathrm{HbA1c}$ was noted not only in patients with poorly controlled type 1 diabetes, but also in well-controlled subjects. The superiority of RT-CGM over SBGM in lowering HbA1c was also confirmed in pump users. Further age-related studies are needed to evaluate the efficacy of this system in the pediatric population, especially in very young children.

\section{Declaration of interest}

A Szypowska and A Ramotowska co-authored educational materials for patients with diabetes, whose edition was sponsored by Abbott. Medtronic MiniMed sponsored the lectures as well as participation in medical conferences. K Dżygało and D Golicki have no conflict of interest.

\section{Funding}

This study was fully funded by the Medical University of Warsaw, Warsaw, Poland.

\section{References}

1 DCCT Study Group. The effect of intensive treatment of diabetes on the development and progression of long-term complications in IDDM. New England Journal of Medicine 1993329 977-986. (doi:10.1056/NEJM199309303291401)

2 Hirsch IB \& Brownlee M. Should minimal blood glucose variability become the gold standard of glycemic control? Journal of Diabetes and its Complications 200519 178-181. (doi:10.1016/j.jdiacomp. 2004.10.001)

3 Jones TW \& Davis EA. Hypoglycemia in children with type 1 diabetes: current issues and controversies. Pediatric Diabetes 2003 4 143-150. (doi:10.1034/j.1399-5448.2003.00025.x)

4 Ives B, Sikes K, Urban A, Stephenson K \& Tamborlane WV. Practical aspects of real-time continuous glucose monitors: the experience of the Yale Children's Diabetes Program. Diabetes Educator 201036 53-62. (doi:10.1177/0145721709352010) 
5 Chetty VT, Almulla A, Odueyungbo A \& Thabane L. The effect of continuous subcutaneous glucose monitoring (CGMS) versus intermittent whole blood finger-stick glucose monitoring (SBGM) on hemoglobin A1c (HBA1c) levels in type I diabetic patients: a systematic review. Diabetes Research and Clinical Practice $2008 \mathbf{8 1}$ 79-87. (doi:10.1016/j.diabres.2008.02.014)

6 Golicki DT, Golicka D, Groele L \& Pankowska E. Continuous glucose monitoring system in children with type 1 diabetes mellitus: a systematic review and meta-analysis. Diabetologia 2008 51 233-240. (doi:10.1007/s00125-007-0884-9)

7 De Block C, Manuel-y-Keenoy B \& Van Gaal LJ. A review of current evidence with continuous glucose monitoring in patients with diabetes. Journal of Diabetes Science and Technology 20082 718-727.

8 Higgins JPT \& Green S (eds). Cochrane Handbook for Systematic Reviews of Interventions 4.2.6 (updated September 2006). The Cochrane Library. Chichester: Wiley, 2007.

9 Robinson KA \& Dickersin K. Development of a highly sensitive search strategy for the retreival of reports of controlled trials using PubMed. International Journal of Epidemiology 200231 150-153. (doi:10.1093/ije/31.1.150)

10 Borenstein M, Hedges L, Higgins J \& Rothstein H. Comprehensive Meta-analysis Version 2. Englewood NJ: Biostat, 2005.

11 Higgins JP, Thompson SG, Deeks JJ \& Altman DG. Measuring inconsistency in metaanalyses. BMJ 2003327 557-560. (doi:10. 1136/bmj.327.7414.557)

12 Juvenile Diabetes Research Foundation Continuous Glucose Monitoring Study Group, Tamborlane WV, Beck RW, Bode BW, Buckingham B, Chase HP, Clemons R, Fiallo-Scharer R, Fox LA, Gilliam LK, Hirsch IB, Huang ES, Kollman C, Kowalski AJ, Laffel L, Lawrence JM, Lee J, Mauras N, O’Grady M, Ruedy KJ, Tansey M, Tsalikian E, Weinzimer S, Wilson DM, Wolpert H, Wysocki T \& Xing D. Continuous glucose monitoring and intensive treatment of type 1 diabetes. New England Journal of Medicine 2008359 1464-1476. (doi:10.1056/NEJMoa0805017)

13 Hirsch IB, Abelseth J, Bode BW, Fischer JS, Kaufman FR, Mastrototaro J, Parkin CG, Wolpert HA \& Buckingham BA. Sensor-augmented insulin pump therapy: results of the first randomized treat-to-target study. Diabetes Technology $\mathcal{E}$ Therapeutics $200810377-383$. (doi:10.1089/dia.2008.0068)

14 Raccah D, Sulmont V, Reznik Y, Guerci B, Renard E, Hanaire H, Jeandidier $\mathrm{N} \&$ Nicolino $\mathrm{M}$. Incremental value of continuous glucose monitoring when starting pump therapy in patients with poorly controlled type 1 diabetes: the RealTrend study. Diabetes Care 200932 2245-2250. (doi:10.2337/dc090750)
15 Juvenile Diabetes Research Foundation Continuous Glucose Monitoring Study Group. The effect of continuous glucose monitoring in well-controlled type 1 diabetes. Diabetes Care 200932 1378-1383. (doi:10.2337/dc09-0108)

16 Kordonouri O, Pankowska E, Rami B, Kapellen T, Coutant R, Hartmann R, Lange K, Knip M \& Danne T. Sensor-augmented pump therapy from the diagnosis of childhood type 1 diabetes: results of the Paediatric Onset Study (ONSET) after 12 months of treatment. Diabetologia 201053 2487-2495. (doi:10.1007/ s00125-010-1878-6)

17 Peyrot M \& Rubin RR. Patient-reported outcomes for an integrated real-time continuous glucose monitoring/insulin pump system. Diabetes Technology \& Therapeutics 200911 57-62. (doi:10.1089/ dia.2008.0002)

18 O'Connell MA, Donath S, O'Neal DN, Colman PG, Ambler GR, Jones TW, Davis EA \& Cameron FJ. Glycaemic impact of patient-led use of sensor-guided pump therapy in type 1 diabetes: a randomised controlled trial. Diabetologia 200952 1250-1257. (doi:10.1007/s00125-009-1365-0)

19 Hoeks LBEA, Greven WL \& de Valk HW. Real-time continuous glucose monitoring system for treatment of diabetes: a systematic review. Diabetic Medicine 201128 386-394. (doi:10.1111/j. 1464-5491.2010.03177.x)

20 Pankowska E, Blazik M, Dziechciarz P, Szypowska A \& Szajewska H. Continuous subcutaneous insulin infusion vs. multiple daily injections in children with type 1 diabetes: a systematic review and meta-analysis of randomized control trials. Pediatric Diabetes 200910 52-58. (doi:10.1111/j.13995448.2008.00440.x)

21 Misso ML, Egberts KJ, Page M, O'Connor D \& Shaw J. Continuous subcutaneous insulin infusion (CSII) versus multiple insulin injections for type 1 diabetes mellitus. Cochrane Database of Systematic Reviews 201020 CDO05103. (doi:10.1002/14651858. CD005103.pub2)

22 Standl E, Schnell O \& Ceriello A. Postprandial hyperglycemia and glycemic variability: should we care? Diabetes Care 201134 S120-S127. (doi:10.2337/dc11-s206)

23 Rewers M, Pihoker C, Donaghue K, Hanas R, Swift P \& Klingensmith GJ. Assessment and monitoring of glycemic control in children and adolescents with diabetes. Pediatric Diabetes 2009 10 71-81. (doi:10.1111/j.1399-5448.2009.00582.x)

24 American Diabetes Association. Standards of Medical Care in Diabetes - 2011. Diabetes Care 201134 S11-S61. (doi:10.2337/dc11-S011)

Received 24 July 2011

Revised version received 7 November 2011

Accepted 17 November 2011 